\title{
Diabetes Mellitus Tipo 2, Depressão e Alterações do Comportamento Alimentar em Doentes Submetidos a Cirurgia Bariátrica
}

\section{Type 2 Diabetes Mellitus, Depression and Eating Disorders in Patients Submitted to Bariatric Surgery}

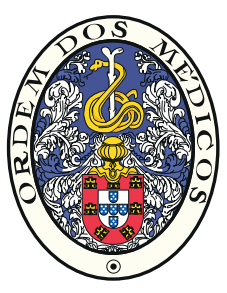

\author{
Isabel BRANDÃO ${ }^{1}$, Ana Marques PINHO², Filipa ARROJADOํ, Ana PINTO-BASTOS ${ }^{3}$, José Maia da COSTA 4 , \\ Rui COELHO ${ }^{1}$, Conceição CALHAU ${ }^{5}$, Eva CONCEIÇÃO $\square^{3}$ \\ Acta Med Port 2016 Mar;29(3):176-181 - http://dx.doi.org/10.20344/amp.6399
}

\section{RESUMO}

Introdução: A obesidade associa-se a um elevado número de comorbilidades, entre as quais a diabetes mellitus tipo 2 e a patologia psiquiátrica. A cirurgia bariátrica tem demonstrado ser a melhor solução para a perda de peso e a melhoria das complicações nos casos de obesidade mórbida. O objetivo deste estudo é o de analisar a evolução da diabetes mellitus tipo 2 e de variáveis psicopatológicas antes e depois da cirurgia bariátrica e verificar o seu impacto na perda de peso.

Material e Métodos: É um estudo longitudinal, que avalia 75 indivíduos antes e depois de serem submetidos a cirurgia bariátrica (47 - banda gástrica; 19 - bypass Roux-en-Y gástrico; 9 - sleeve gástrico), com tempo de follow-up entre 18 e 46 meses. Os instrumentos de avaliação foram a entrevista clínica e os questionários Eating Disorder Examination questionnaire - EDE-Q e Beck Depression Inventory - BDI.

Resultados: Verificou-se melhoria relativamente à variável dicotómica diabetes mellitus tipo 2 após a cirurgia $\left(\chi^{2}(1)=26,132, p<\right.$ 0,001). Não ocorreu melhoria significativa em termos de patologia psiquiátrica quando a análise foi controlada para o tipo de cirurgia. Encontrámos uma associação significativa entre as variáveis em estudo no pós-cirúrgico e a perda de peso. Este modelo explica $27 \%$ da variação do peso após a cirurgia $\left(R^{2}=0,265\right)$ e é significativo $F(3,33)=2,981, p=0,038$.

Discussão: A diabetes mellitus tipo 2, patologia depressiva ou do comportamento alimentar mostraram estar relacionados com a perda de peso. Não ficou esclarecido em que sentido esta relação é estabelecida, nem a relação que estas variáveis metabólicas e psicológicas possam ter entre si ao longo do período pós cirurgia bariátrica.

Conclusão: A diabetes mellitus tipo 2 evoluiu favoravelmente após a cirurgia. Diabetes mellitus tipo 2, depressão e patologia alimentar no período pós-cirúrgico associaram-se a menor perda de peso. As variáveis estudadas no período pré cirúrgico não contribuíram de forma significativa para a perda de peso.

Palavras-chave: Cirurgia Bariátrica; Comportamento Alimentar; Depressão; Diabetes Mellitus Tipo 2; Obesidade Mórbida.

\section{ABSTRACT}

Introduction: Obesity is associated with a great number of complications, including type 2 diabetes mellitus and psychiatric pathology. Bariatric surgery is the best solution to weight loss and improvement of complications in morbid obese patients. This study aims to analyze the evolution of type 2 diabetes mellitus and psychopathologic variables before and after bariatric surgery and assess the importance of different variables in weight loss.

Material and Methods: This is a longitudinal study, which evaluates 75 patients before and after bariatric surgery (47 - LAGB laparoscopic adjustable gastric band; 19 - RYGB - Roux-en-Y gastric bypass; 9 - sleeve) with a follow-up time between 18 and 46 months. A clinical interview and self report questionnaires were applied - Eating Disorder Examination questionnaire - EDE-Q and Beck Depression Inventory - BDI.

Results: Results show an improvement in type 2 diabetes mellitus after surgery $\left(\chi^{2}(1)=26.132, p<0.001\right)$. There was not a significant improvement among psychiatric pathology when we controlled the analysis for the type of surgery. It was verified that type 2 diabetes mellitus, depression and eating disorders in post-operative period are associated with less weight loss. This model explains $27 \%$ of weight variance after surgery $\left(R^{2}=0.265\right)$ and it is significant $F(3.33)=2.981, p=0.038$.

Discussion: Type 2 diabetes mellitus, psychiatric pathology and eating disorders after surgery influenced weight loss. It was not clear in what way this relation was verified, neither the relation that these metabolic and psychological variables may have during the postoperative period.

Conclusion: Type 2 diabetes mellitus improved after surgery. Type 2 diabetes mellitus, depression and eating disorders influenced weight loss in the postoperative period. These variables did not influence weight loss in the preoperative period.

Keywords: Bariatric Surgery; Depressive Disorder; Diabetes Mellitus, Type 2; Feeding and Eating Disorders; Obesity, Morbid.

\section{INTRODUÇÃO}

A obesidade define-se como um excesso de gordura corporal sendo avaliada pelo Índice de Massa Corporal (IMC) se igual ou superior a $30 \mathrm{~kg} / \mathrm{m}^{2}$ e classificada como

obesidade mórbida quando o IMC é igual ou superior a 40 $\mathrm{kg} / \mathrm{m}^{2}$.

É uma patologia preocupante devido ao crescente

1. Departamento de Neurociências Clínicas e Saúde Mental. Faculdade de Medicina. Universidade do Porto. Centro Hospitalar de São João. Porto. Portugal.

2. Faculdade de Medicina. Universidade do Porto. Porto. Portugal.

3. Escola de Psicologia. Centro de Investigação em Psicologia. Universidade do Minho. Braga. Portugal.

4. Serviço de Cirurgia. Hospital de Braga. Braga. Portugal.

5. Departamento de Bioquímica. Faculdade de Medicina. Universidade do Porto. Porto. Portugal.

$\triangle$ Autor correspondente: Eva Conceição. econceicao@psi.uminho.pt

Recebido: 13 de março de 2015 - Aceite: 28 de setembro de 2015 | Copyright @ Ordem dos Médicos 2016 
aumento do número de casos bem como das diversas comorbilidades a que está associada. Nos últimos 20 anos duplicaram os casos de obesidade mórbida, sendo estimada a existência de 400 milhões de obesos no Mundo., ${ }^{1,2}$

Em Portugal, a sua prevalência tem vindo a aumentar, sendo que, entre 2003 e 2005, 52,4\% da população portuguesa era obesa ou tinha excesso de peso, números que têm vindo a crescer. ${ }^{3,4}$

Este problema de saúde pública está associado ao dobro da mortalidade e a um elevado número de comorbilidades, nomeadamente diabetes mellitus tipo 2, patologia psicológica ou psiquiátrica (como depressão, ansiedade ou perturbações alimentares), patologia cardiovascular e cancro. As comorbilidades associadas à obesidade mórbida são em grande parte responsáveis pelo seu mau prognóstico e pelo aumento dos custos associados à saúde. 1,5,6

A cirurgia bariátrica tem vindo a apresentar-se como o método mais eficaz para a obtenção de resultados satisfatórios em termos de perda de peso, melhoria das comorbilidades e aumento da sobrevida, estando indicada para doentes com obesidade mórbida (IMC > $40 \mathrm{~kg} / \mathrm{m}^{2}$ ) ou IMC superior a $35 \mathrm{~kg} / \mathrm{m}^{2}$ na condição de ocorrerem importantes comorbilidades associadas. ${ }^{5-10}$ Está também descrita uma melhoria geral da qualidade de vida dos pacientes submetidos a este procedimento. ${ }^{11,12}$ Contudo, os resultados obtidos após este tipo de intervenção não são uniformes, existindo casos em que a perda de peso é insatisfatória, pelo que se tem tentado estudar quais os fatores relacionados com uma menor perda de peso em alguns doentes. ${ }^{13,17}$

Dos diferentes tipos de procedimentos realizados na cirurgia bariátrica há dois efeitos preponderantes: os mal absortivos e os de restrição. As cirurgias de mal absorção (como o bypass gástrico) permitem, através do encurtamento do trato gastrointestinal, uma diminuição do período de digestão e absorção de nutrientes, resultando num balanço de energia negativo e perda de peso. Os procedimentos restritivos (de que são exemplo a banda gástrica e parcialmente o sleeve gástrico) atuam devido a uma redução da capacidade de armazenamento do estômago, levando a um menor aporte calórico. Existem ainda métodos mistos, como o bypass Roux-en-Y.6,7

Como referido anteriormente, os candidatos a cirurgia bariátrica têm frequentemente problemas metabólicos como a diabetes mellitus tipo 2. A literatura demonstra que existe uma melhoria significativa no que concerne a esta patologia após a realização de cirurgia bariátrica. ${ }^{6,11,18,19}$

A literatura apoia também a hipótese de que os indivíduos obesos, nomeadamente os candidatos a cirurgia bariátrica, têm mais frequentemente patologia do foro psicológico/psiquiátrico relativamente a indivíduos não obesos; 20 a $60 \%$ dos doentes sofrem de doença psiquiátrica do eixo I, ${ }^{20}$ sendo a depressão, a ansiedade e o binge eating

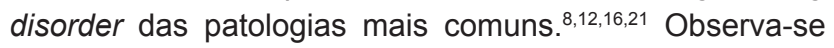
também uma associação entre fatores psicológicos e psicossociais, comportamento alimentar disfuncional e obesidade. ${ }^{13,14,21,22}$

Em termos de evolução após cirurgia bariátrica, os estudos não são unânimes relativamente a este tópico, sendo que em alguns deles não são referidas melhorias significativas em termos de saúde mental. ${ }^{10}$ Noutros casos, verificaram-se melhorias em termos de patologia depressiva ou ansiosa, com melhores resultados para a depressão. ${ }^{12,18,19,23}$

A diabetes mellitus tipo 2 e as perturbações do foro psicológico/psiquiátrico são problemas que ocorrem em simultâneo e que se poderão relacionar entre si, sendo importante compreender a sua evolução após realização de cirurgia bariátrica e a sua influência nos resultados da cirurgia. ${ }^{12,24,25}$ Tem sido demonstrado que tanto o comportamento alimentar ${ }^{14,22}$ como a depressão $0^{9,13}$ no período pós-cirúrgico se associam a uma menor perda de peso. ${ }^{8} \mathrm{Um}$ estudo recente demonstrou a associação entre aumento de peso após a cirurgia e episódios de ingestão alimentar compulsiva, preocupações com a imagem corporal e sintomas depressivos. ${ }^{26}$ Também a existência de diabetes mellitus tipo 2 tem vindo a ser associada a piores resultados de tratamento. No entanto, variáveis médicas e psicológicas são tradicionalmente avaliadas de forma independente e são reduzidos os estudos em que o impacto de fatores psicossociais é avaliado em conjunto com variáveis médicas no mesmo modelo de compreensão da sua possível relação com a perda de peso..$^{21,27,28}$

O objetivo deste estudo é, então, o de avaliar a evolução da diabetes mellitus tipo 2 e das variáveis psicopatológicas (depressão e patologia alimentar) antes e depois da realização de cirurgia bariátrica, bem como analisar a importância das diferentes variáveis nos resultados de perda de peso, testando um modelo integrado que inclua variáveis médicas e psicológicas.

\section{MATERIAL E MÉTODOS}

O presente estudo é do tipo observacional e longitudinal de caráter retrospetivo e analisa dados de 75 indivíduos, antes e depois de serem submetidos a cirurgia bariátrica (47 - banda gástrica; 19 - bypass gástrico - RYGB; 9 - sleeve gástrico), em dois Hospitais distintos: Centro Hospitalar de São João, no Porto, ( $n=56 ; 74,7 \%$ ) e Hospital de Braga $(n=19 ; 25,3 \%)$. A recolha de dados foi efetuada entre janeiro de 2009 e junho de 2013. Os indivíduos tinham de reunir os seguintes critérios de inclusão: serem candidatos a cirurgia bariátrica; terem preenchido os questionários de autorrelato a seguir explicitados; terem sido observados durante um tempo de follow-up mínimo de 18 meses. Definiram-se como critérios de exclusão os doentes terem sido submetidos a cirurgia subsequente ou a ocorrência de gravidez durante o período de follow-up. Foi pedida aprovação pela comissão de ética das instituições envolvidas e todos os participantes preencheram consentimento informado.

Para avaliação dos sujeitos, utilizámos uma entrevista semiestruturada e questionários de autorrelato (Beck Depression Inventory - BDI e Eating Disorder Examination questionnaire - EDE-Q), tendo a entrevista sido realizada por psicólogo experiente neste tipo de avaliação. Os participantes eram avaliados antes da cirurgia e depois da 
cirurgia, sendo que o tempo de follow-up variou entre os 18 e os 46 meses.

\section{Instrumentos}

\section{Entrevista clínica de acordo com a DSM-IV20}

Inclui dados que permitem determinar a caracterização sociodemográfica, tipo de cirurgia, tempo decorrido desde a intervenção e antecedentes clínicos relevantes. Os dados clínicos de diagnóstico e metabólicos foram retirados dos registos nos processos dos Hospitais.

\section{Eating Disorder Examination questionnaire (EDE-Q) $)^{29,30}$}

Consiste num inquérito de autorrelato de 36 itens cujo objetivo é o de estimar a frequência e gravidade de comportamentos alimentares patológicos num espaço de 28 dias. A fidelidade e consistência deste método têm vindo a ser reconhecidas, sendo que em Portugal se verifica igualmente a validade do uso destes questionários. O objetivo da aplicação destes inquéritos é assim o de averiguar a existência de patologia alimentar.

\section{Beck Depression Inventory (BDI) $)^{31,32}$}

Consiste num inquérito cujo objetivo é o de estimar a existência de sintomas depressivos nos últimos sete dias. Deste questionário fazem parte 21 perguntas de escolha múltipla, sendo cada pergunta avaliada de 0 a 3 , num total cujo valor máximo é de 63 . Tem sido demonstrado que este é um método fiável e aplicável também à população portuguesa.

\section{Diagnóstico de diabetes mellitus tipo 2}

O diagnóstico de diabetes mellitus tipo 2 foi confirmado por médico endocrinologista de acordo com os critérios de diagnóstico vigentes na norma da Direção Geral de Saúde. ${ }^{33}$

\section{Análises estatísticas}

Inicialmente utilizámos um teste de Qui-quadrado para avaliar a evolução da variável dicotómica 'diabetes mellitus tipo 2', antes e depois da realização de cirurgia bariátrica. Utilizámos também um teste de Modelo Linear Generalizado (ANOVA) para medidas repetidas de forma a analisar diferenças entre os dois momentos de avaliação nas variáveis psiquiátricas selecionadas para o estudo. As análises foram controladas para o tipo de cirurgia.

De forma a compreender o papel das variáveis em estudo na perda de peso, realizámos uma análise de Regressão Linear Múltipla. Deste modo, foi testada a influência das diferentes variáveis (diabetes mellitus tipo 2, depressão, e perturbações alimentares) no período pré-cirúrgico e no período pós-cirúrgico na perda de peso. A variável perda de peso foi definida pela percentagem de perda do peso em excesso desde a cirurgia até ao momento da avaliação.

Procedemos à análise dos dados recolhidos no programa IBM SPSS, Versão 20. Considerámos existir significância estatística para um valor de $p<0,05$. Ocorreram diferenças no tamanho da amostra devido a missing values.

\section{RESULTADOS}

A amostra foi constituída por 75 participantes. Os sujeitos encontravam-se entre os 23 e os 64 anos de idade, sendo 64 participantes $(85,3 \%)$ do género feminino e 11 participantes $(14,7 \%)$ do género masculino. Destes, 47 $(62,7 \%)$ foram submetidos a colocação de banda gástrica, $19(25,3 \%)$ foram submetidos a bypass Roux-en-Y gástrico (RYGB) e nove (12\%) foram submetidos a sleeve gástrico A Tabela 1 resume as características sociodemográficas da amostra. A Tabela 2 resume a informação relativa à evolução do IMC após o procedimento bem como os valores da percentagem de peso em excesso perdida.

Relativamente à evolução da diabetes mellitus tipo 2 , os resultados revelaram que, na fase pré-cirurgia, $50,7 \%$ dos indivíduos $(n=34)$ não eram diabéticos. Na fase pós-cirurgia, 61,2\% ( $n=41)$ dos indivíduos encontravam-se sem doença. Verificou-se uma diferença significativa entre as fases pré e pós cirurgia, $\chi^{2}(1)=26,132, p<0,001$. A proporção de doentes diabéticos que melhorou após a cirurgia foi estatisticamente significativa. A Tabela 3 resume a evolução da doença nesta amostra.

Em termos de evolução de patologia psiquiátrica, verificou-se que ocorreu uma melhoria estatisticamente significativa em termos de depressão (Wilk's Lambda $=0,89$, $F=1,38, p=0,006)$. Também a variável patologia alimentar apresentou melhoria após a cirurgia (Wilk's Lambda = $0,806, F=1,797, p=0,008)$. Quando controlada para o tipo de cirurgia, a análise não demonstrou diferenças estatisticamente significativas.

Para compreender o papel das diferentes variáveis (diabetes mellitus tipo 2, depressão - BDI e patologia alimentar - EDE-Q) na perda de peso, realizaram-se dois testes de Regressão Linear Múltipla, o primeiro utilizando as variáveis relativas ao período pré-cirurgia e o segundo estudando as variáveis relativas ao período pós-cirúrgico.

Tabela 1 - Características sociodemográficas da amostra

\begin{tabular}{|c|c|}
\hline \multicolumn{1}{|c|}{ Género } & $64(85,3 \%)$ \\
\hline \multicolumn{1}{|c|}{ Feminino } & $11(14,7 \%)$ \\
\hline Idade & \\
\hline \multicolumn{1}{|c|}{ Mínima } & 23 anos \\
\hline Máxima & 64 anos \\
\hline Tempo de follow-up & 18 meses \\
\hline Mínimo & 46 meses \\
\hline Máximo & \\
\hline Tipo de procedimento & $47(62,7 \%)$ \\
\hline Banda gástrica & $19(25,3 \%)$ \\
\hline Bypass gástrico Roux-en-Y & $9(12,0 \%)$ \\
\hline Sleeve gástrico & $\mathrm{n}=75$ \\
\hline
\end{tabular}


Tabela 2 - Evolução do IMC e percentagem de peso em excesso perdido

\begin{tabular}{|c|c|}
\hline \multicolumn{2}{|c|}{ IMC pré-cirurgia } \\
\hline Mínimo & $34,53 \mathrm{~kg} / \mathrm{m}^{2}$ \\
\hline Máximo & $59,82 \mathrm{~kg} / \mathrm{m}^{2}$ \\
\hline Médio & $44,75 \mathrm{~kg} / \mathrm{m}^{2}$ \\
\hline \multicolumn{2}{|c|}{ IMC pós-cirurgia } \\
\hline Mínimo & $21,45 \mathrm{~kg} / \mathrm{m}^{2}$ \\
\hline Máximo & $57,26 \mathrm{~kg} / \mathrm{m}^{2}$ \\
\hline Médio & $32,79 \mathrm{~kg} / \mathrm{m}^{2}$ \\
\hline \multicolumn{2}{|c|}{$\%$ de peso em excesso perdido } \\
\hline Mínimo & $-2,44 \%$ (ganho de peso) \\
\hline Máximo & $105,44 \%$ \\
\hline Médio & $55,56 \%$ \\
\hline
\end{tabular}

Relativamente ao primeiro teste, constatámos que as variáveis no período pré-cirúrgico não contribuíram de forma significativa para a perda de peso $(\mathrm{F}(3,39)=0,55, p=$ 0,65).

Pelo contrário, as variáveis relativas ao período pós-cirúrgico mostraram associar-se significativamente a perda de peso. Segundo o teste de regressão linear múltipla, e sendo a variável dependente a perda de peso e as variáveis independentes a diabetes mellitus tipo 2, a patologia alimentar (EDE-Q) e a depressão (BDI), verificou-se uma associação significativa entre estes fatores e a perda de peso. De acordo com os resultados este modelo explica $27 \%$ da variação do peso após a cirurgia $\left(R^{2}=0,265\right)$ e é significativo $F(3,33)=2,981, p=0,038$. A diabetes mellitus tipo 2 foi a variável que demonstrou associar-se de forma mais relevante a perda de peso $(\beta=-0,484, t=-2,373, p=$ $0,024)$. A patologia alimentar $(\beta=-0,1, t=-0,38, p=0,341)$ e a depressão $(\beta=-0,249, t=-0,967, p=0,341)$ contribuíram também, ainda que de forma marginalmente significativa, para a perda de peso.

\section{DISCUSSÃO}

Neste estudo, verificámos uma melhoria estatisticamente significativa em termos da diabetes mellitus tipo 2 após cirurgia bariátrica, o que está de acordo com o referido na literatura. ${ }^{6,11,18,19}$ Contudo, o tempo de follow-up destes doentes é ainda relativamente curto (mínimo de 18 meses, máximo de 46 meses), sendo que seria fundamental manter o acompanhamento dos doentes de forma a analisar a evolução ao longo do tempo - existe inclusivamente um artigo que refere que a resolução da diabetes mellitus tipo 2 é três vezes mais frequente aos 10 anos comparativamente a métodos de intervenção não cirúrgicos. ${ }^{34}$
Constatámos que as variáveis psicopatológicas utilizadas neste estudo evoluíram favoravelmente após intervenção cirúrgica, mas apenas quando a análise não foi controlada para o tipo de cirurgia. Este aspeto relaciona-se possivelmente com o facto de a melhoria sintomática estar associada principalmente à realização de bypass gástrico, possivelmente por implicar perdas de peso mais acentuadas, menos efeitos laterais associados, maior grau de satisfação com o procedimento ou outras condicionantes.

Estudos anteriormente realizados sugerem que a realização de cirurgia bariátrica em doentes obesos favorece a melhoria em termos de patologia psiquiátrica. ${ }^{12,18,19,23}$ Contudo, esta é uma área de conhecimento que necessita de maior investigação porque estes resultados não são consistentes entre os diferentes estudos. ${ }^{11}$

A existência de diabetes mellitus tipo 2 no período pós-cirurgia associou-se a uma menor percentagem de peso perdido (sendo, das variáveis analisadas, aquela que mais impacto teve na perda de peso). Como referido, a variável em análise não influencia a perda de peso quando presente no período pré-cirúrgico. De facto, há na literatura evidência de que a resolução da diabetes mellitus tipo 2 está relacionada com a perda de peso após a cirurgia, pelo que a não resolução desta patologia poderá contribuir para uma menor perda de peso. ${ }^{35}$

A relação da patologia psiquiátrica com o peso perdido é controversa. De acordo com este estudo, a depressão foi uma das variáveis que integrou o modelo associado a perda de peso. A literatura demonstra que o impacto do estado psiquiátrico nos resultados de perda de peso após cirurgia não é claro. ${ }^{21}$ De facto, o funcionamento psicossocial e as doenças psiquiátricas no período pós cirúrgico podem, por um lado, influenciar a perda de peso, e por outro, serem influenciados por esse resultado. ${ }^{12,16}$ Com este estudo não foi possível esclarecer se o facto de os doentes se encontrarem num pior estado psicológico levou a uma menor perda de peso ou se foi o facto de não terem obtido o resultado pretendido que contribuiu para um pior estado em termos de saúde mental.

Outro resultado obtido foi o de que a existência de perturbações alimentares no período pós-cirurgia se relaciona com a percentagem de peso perdido, o que está de acordo com a literatura. Efetivamente concluiu-se em vários estudos que a existência de perturbações alimentares no pós-cirúrgico prediz um pior resultado (menos perda de peso), mas não no pré-cirúrgico, tal como se verificou neste caso. ${ }^{12,17}$

O facto de este ser um estudo longitudinal confere robustez aos nossos resultados. No entanto, possui limitações que importa referir. De forma a melhor sustentar os dados obtidos, teria sido vantajosa uma maior uniformidade em relação ao tipo de cirurgia efetuado, assim como

Tabela 3 - Evolução da diabetes mellitus tipo 2

\begin{tabular}{|c|c|c|}
\hline Pré-cirurgia & $50,7 \%(n=34)$ não diabéticos & $49,3 \%(n=33)$ diabéticos \\
\hline Pós-cirurgia & $61,2 \%(n=41)$ não diabéticos & $38,8 \%(n=26)$ diabéticos \\
\hline
\end{tabular}


proveitosa a obtenção de uma maior amostra de sujeitos, bem como um tempo de follow-up mais alargado. Além disso, convém lembrar que o estudo se baseou em questionários de autoavaliação, o que se pode traduzir em viés (como por exemplo viés de memória ou de classificação no que concerne ao diagnóstico da psicopatologia).

\section{CONCLUSÃO}

Com este estudo verificámos que, após a realização de cirurgia bariátrica, existe efetivamente uma melhoria em termos de diabetes mellitus tipo 2. Constatámos ainda que os fatores diabetes mellitus tipo 2, depressão e patologia alimentar influenciam a perda de peso no período pós-cirúrgico.

Os resultados obtidos ressalvam a importância de um seguimento continuado pela Psiquiatria em doentes submetidos a cirurgia bariátrica. Assim sendo, urge a necessidade de criar estratégias individualizadas em termos de acompanhamento psicológico/psiquiátrico de modo a aumentar o sucesso obtido com a cirurgia bariátrica.

A avaliação da depressão e sobretudo das alterações do comportamento alimentar nos doentes submetidos a cirurgia bariátrica deve ter em conta o tipo de cirurgia escoIhido.

Será importante a realização de estudos posteriores de forma a corrigir as limitações referidas neste trabalho, bem como investigar novas variáveis que possam ter um papel preponderante na perda de peso, influenciando assim o sucesso destas intervenções.

\section{REFERÊNCIAS}

1. Al-Hadithy N, Mennie J, Magos T, Stewart K. Desire for post bariatric body contouring in South East Scotland. J Plast Reconstr Aesthet Surg. 2013;66:87-94.

2. World Health Organization. Obesity: preventing and managing the global epidemic. Report of a WHO consultation. World Health Organ Tech Rep Ser. 2000;894:1-25.

3. do Carmo I, Dos Santos O, Camolas J, Vieira J, Carreira M, Medina L, et al. Overweight and obesity in Portugal: national prevalence in 20032005. Obes Rev. 2008;9:11-9.

4. do Carmo I, dos Santos O, Camolas J, Vieira J, Carreira M, Medina L, et al. Prevalence of obesity in Portugal. Obes Rev. 2006;7:233-7.

5. Woodman G, Cywes R, Billy H, Montgomery K, Cornell C, Okerson T. Effect of adjustable gastric banding on changes in gastroesophageal reflux disease (GERD) and quality of life. Curr Med Res Opin. 2012;28:581-9.

6. Cannon CP, Kumar A. Treatment of overweight and obesity: lifestyle, pharmacologic, and surgical options. Clin Cornerstone. 2009;9:55-68.

7. Bariatric surgery for people with diabetes and morbid obesity: an evidence-based analysis. Ont Health Technol Assess Ser. 2009;9:1-23.

8. Gradaschi R, Noli G, Cornicelli M, Camerini G, Scopinaro N, Adami GF. Do clinical and behavioural correlates of obese patients seeking bariatric surgery differ from those of individuals involved in conservative weight loss programme? J Hum Nutr Diet. 2013;26:S34-8.

9. Junior WS, do Amaral JL, Nonino-Borges CB. Factors related to weight loss up to 4 years after bariatric surgery. Obes Surg. 2011;21:1724-30.

10. Kaul A, Sharma J. Impact of bariatric surgery on comorbidities. Surg Clin North Am. 2011;91:1295-312.

11. Julia C, Ciangura C, Capuron L, Bouillot JL, Basdevant A, Poitou C, et al. Quality of life after Roux-en-Y gastric bypass and changes in body mass index and obesity-related comorbidities. Diabetes Metab J. 2013;39:148-54.

12. de Zwaan M, Enderle J, Wagner S, Muhlhans B, Ditzen B, Gefeller O, et al. Anxiety and depression in bariatric surgery patients: a prospective, follow-up study using structured clinical interviews. J Affect Disord.

\section{AGRADECIMENTOS}

Os autores gostariam de agradecer a Gil Faria (Centro Hospitalar de São João, Porto) pela colaboração na recolha de dados para o estudo.

\section{PROTECÇÃO DE PESSOAS E ANIMAIS}

Os autores declaram que os procedimentos seguidos estavam de acordo com os regulamentos estabelecidos pelos responsáveis da Comissão de Investigação Clínica e Ética e de acordo com a Declaração de Helsínquia da Associação Médica Mundial.

\section{CONFIDENCIALIDADE DOS DADOS}

Os autores declaram ter seguido os protocolos do seu centro de trabalho acerca da publicação dos dados de doentes.

\section{CONFLITO DE INTERESSES}

Os autores declaram não ter nenhum conflito de interesses relativamente ao presente artigo.

\section{FONTES DE FINANCIAMENTO}

Esta investigação foi parcialmente suportada pela Fundação para a Ciência e Tecnologia, Portugal através do projeto (PTDC/MHC-PCL/4974/2012) e bolsa (SFRH/ BPD/78896/2011) concedidos a Eva Conceição, e bolsa (SFRH/BD/104159/2014) concedida a Ana Pinto-Bastos.

2011;133:61-8.

13. Sousa P, Bastos AP, Venancio C, Vaz AR, Brandao I, Costa JM, et al. Compreender a sintomatologia depressiva após a cirurgia bariátrica: o papel do peso, da alimentação e da imagem corporal. Acta Med Port. 2014;27:450-7.

14. Conceição E, Bastos AP, Brandao I, Vaz AR, Ramalho S, Arrojado F, et al. Loss of control eating and weight outcomes after bariatric surgery: a study with a Portuguese sample. Eat Weight Disord. 2014;19:103-9.

15. Sjostrom CD. Surgery as an intervention for obesity. Results from the Swedish obese subjects study. Growth Horm IGF Res. 2003;13:S22-6.

16. Mitchell JE, Crosby R, de Zwaan M, Engel S, Roerig J, Steffen K, et al. Possible risk factors for increased suicide following bariatric surgery. Obesity. 2013;21:665-72.

17. Kinzl JF. Morbid obesity: significance of psychological treatment after bariatric surgery. Eat Weight Disord. 2010;15:e275-80.

18. Behrens C, Tang BQ, Amson BJ. Early results of a Canadian laparoscopic sleeve gastrectomy experience. Can J Surg. 2011;54:138-43.

19. Mathus-Vliegen EM, de Weerd S, de Wit LT. Health-related quality-oflife in patients with morbid obesity after gastric banding for surgically induced weight loss. Surgery. 2004;135:489-97.

20. DSM-IV - (1994). American Psychiatric Association. Diagnostic and statistical manual of mental disorders. $4^{\text {th }}$ ed. Washington: American Psychiatric Association; 1994

21. Sarwer DB, Wadden TA, Fabricatore AN. Psychosocial and behavioral aspects of bariatric surgery. Obes Res. 2005;13:639-48.

22. Meany G, Conceição E, Mitchell JE. Binge eating, binge eating disorder and loss of control eating: effects on weight outcomes after bariatric surgery. Eur Eat Disord Rev. 2014;22:87-91.

23. Assimakopoulos K, Karaivazoglou K, Panayiotopoulos S, Hyphantis T, Iconomou G, Kalfarentzos F. Bariatric surgery is associated with reduced depressive symptoms and better sexual function in obese female patients: a one-year follow-up study. Obes Surg. 2011;21:362-6.

24. Webb JB, Applegate KL, Grant JP. A comparative analysis of Type 2 diabetes and binge eating disorder in a bariatric sample. Eat Behav. 
2011; $12: 175-81$

25. Zhang J, Xu CP, Wu HX, Xue XJ, Xu ZJ, Li Y, et al. Comparative study of the influence of diabetes distress and depression on treatment adherence in Chinese patients with type 2 diabetes: a cross-sectional survey in the People's Republic of China. Neuropsychiatr Dis Treat. 2013;9:1289-94.

26. Ramalho S, Bastos AP, Silva C, Vaz AR, Brandao I, Machado PP, et al. Excessive skin and sexual function: relationship with psychological variables and weight regain in women after bariatric surgery. Obes Surg. 2014.

27. Clark MM, Balsiger BM, Sletten CD, Dahlman KL, Ames G, Williams $\mathrm{DE}$, et al. Psychosocial factors and 2-year outcome following bariatric surgery for weight loss. Obes Surg. 2003;13:739-45.

28. Sutton D, Raines DA. Health-related quality of life following a surgical weight loss intervention. Appl Nurs Res. 2010;23:52-6.

29. Fairburn CG, Beglin SJ. Assessment of eating disorders: interview or self-report questionnaire? Int J Eat Disord. 1994;16:363-70.

30. Machado PP, Martins C, Vaz AR, Conceição E, Bastos AP, Goncalves S.
Eating disorder examination questionnaire: psychometric properties and norms for the Portuguese population. Eur Eat Disord Rev. 2014;22:44853.

31. Wang YP, Gorenstein C. Psychometric properties of the Beck Depression Inventory-II: a comprehensive review. Rev Bras Psiquiatr. 2013;35:416-31.

32. Vaz Serra A, Pio Abreu J. Aferição dos quadros clínicos depressivos I-Ensaio de aplicação do "Inventário Depressivo de Beck" a uma amostra portuguesa de doentes deprimidos. Coimbra MED 1973; XX:623-644

33. Boavida JM, Duarte A, Vicente LF, Ruas MA, Melo PC. Norma 002/2011 Diagnóstico e Classificação de Diabetes Mellitus. Lisboa: DGS; 2011.

34. Sjostrom L, Lindroos AK, Peltonen M, Torgerson J, Bouchard C Carlsson B, et al. Lifestyle, diabetes, and cardiovascular risk factors 10 years after bariatric surgery. N Engl J Med. 2004;351:2683-93.

35. Buchwald H, Estok R, Fahrbach K, Banel D, Jensen MD, Pories WJ, et al. Weight and type 2 diabetes after bariatric surgery: systematic review and meta-analysis. Am J Med. 2009;122:248-56.e5. 